\title{
PREDAÇÃO DE SEMENTES DE LEGUMINOSAS POR BRUQUIDEOS (INSECTA: COLEOPTERA) NA SERRA DOS CARAJÁS, PARÁ, BRASIL ${ }^{1}$
}

Recebido 24.10.91. Aceito 09.06.94.

\author{
Cecília Lomônaco²
}

\begin{abstract}
RESUMO: (Predação de sementes de leguminosas por bruquídeos (Insecta: Coleoptera) na Serra dos Carajás, Pará, Brasil). Estudou-se a predação de sementes em Bauhinia pulchella Benth. (Caesalpiniaceae), Mimosa acutistipula Benth. var. nigra Hub., Mimosa somnians H.B. ex Willd. (Mimosaceae) e Phaseolus linearis H.B.K. (Fabaceae) para investigar a taxa de predação e a existência de defesas contra a ação de predadores. Foi constatada a preferência por sementes de maior tamanho pelo bruquídeo de Bauhinia pulchella, o que pode significar uma adaptação das plantas em ter sementes pequenas que escapem da predação. Em Mimosa somnians, a imprevisibilidade do número de sementes viáveis produzidas poderia consistir num mecanismo de defesa, por impedir a otimização da quantidade de ovos deixados pelo predador em cada fruto. $\mathrm{O}$ formato extremamente achatado das sementes de Mimosa acutistipula parece limitar a ação de predadores. A alta resistência da casca dos frutos de Phaseolus linearis e o aspecto compacto e duro de suas sementes podem ser considerados defesas mecânicas. Existe relação entre o tamanho de sementes e o tamanho de predadores para as espécies estudadas.
\end{abstract}

Palavras-chave: Predação de sementes, Bruchidae, Leguminosae.

\begin{abstract}
Legume seed damage by bruchids (Insecta: Coleoptera) in Serra dos Carajás, Pará State, Brazil). Seed damage in Bauhinia pulchella Benth. (Caesalpiniaceae), Mimosa acutistipula Benth var. nigra Hub., Mimosa somnians H.B. ex Willd. (Mimosaceae) and Phaseolus linearis H.B.K. (Fabaceae), was studied to investigate defense against predators. The preference for larger seeds of Bauhinia pulchella by bruchids is a selection pressure for the plant to product smaller seeds, as a survival mechanism to scape predation. The impredictability of the number of viable seeds per pod in Mimosa somnians could represent a defense mechanism because it does not permit the optimization of the number of eggs laid in each fruit. The flattened seeds of Mimosa acutistipula limit the attack sucess of predator beetles. The high resistance of the pod skin and the hard compact seeds in Phaseolus linearis may be considered mechanical defenses. There is a correlation between seed size and predator size in the species studied.
\end{abstract}

Key Words: Seed predators, Bruchidae, Legumes.

\footnotetext{
'Trabalho realizado durante a disciplina Ecologia de Campo I, do curso de Pós-graduação em Ecologia da Universidade Estadual de Campinas.

2 Departamento de Biociências, Universidade Federal de Uberlândia, Caixa Postal 593. 38400-902, Uberlândia-MG, Brasil.
} 


\section{Introdução}

Besouros Bruchidae são importantes predadores de sementes em grande variedade de plantas (Janzen 1969). A intensidade e o padrão deste tipo de predação podem afetar diretamente a biologia de populações de fanerógamas (Janzen 1970). Vários trabalhos discutem algumas possíveis respostas evolutivas de plantas contra predação (Wilson \& Janzen 1972; Center \& Johnson 1974; Fernandes \& Whithan 1989). Janzen (1971) agrupou as defesas de leguminosas em três principais tipos: a) defesas químicas; b) saciação do predador, em que a produção de sementes é superior à capacidade de consumo dos predadores; e c) imprevisibilidade de recursos, em que a frutificação ocorre em intervalos plurianuais irregulares. Smith (1975) apontou, também, a variabilidade no número e tamanho de sementes produzidas como estratégia antipredação. Defesas vegetais podem, entretanto, tornar-se ineficazes, uma vez que são facilmente contornadas por predadores (Connell 1970).

Neste estudo foi avaliada a taxa de predação de sementes em quatro espécies de leguminosas e verificada a existência de possíveis mecanismos de defesas destas espécies contra a ação de predadores.

\section{Material e métodos}

O estudo foi realizado na área de canga de Serra Norte (5'54'S; 49 53'W), Carajás, Pará, Brasil, durante o mês de julho de 1988. A canga consiste de áreas de afloramento de minério de ferro de $3 \mathrm{~m}$ ou mais de espessura, originando campos colonizados, predominantemente, por vegetação herbácea (canga aberta) e arbustiva (canga densa) (Secco \& Mesquita 1983).

Foram coletados 50 frutos maduros fechados de cada uma das leguminosas estudadas: Bauhinia pulchella Benth (Caesalpiniaceae), Mimosa acutistipula Benth. var. nigra Hub., Mimosa somnians H.B. ex Willd. (Mimosaceae) e Phaseolus linearis H.B.K. (Fabaceae).

As sementes foram retiradas dos frutos e classificadas em viáveis (com desenvolvimento normal) e não viáveis (abortadas), por fruto. Dentre as sementes viáveis, foram contadas as que apresentavam evidência de predação ou de ataque de fungos. $\mathrm{O}$ comprimento das sementes, dos frutos e dos predadores, quando presentes, foi medido com paquímetro (precisão de $0,001 \mathrm{~mm}$ ). O formato e consistência das sementes e a resistência da casca dos frutos foram analisados comparativamente. Foram calculadas as médias, os desvios padrões e os coeficientes de variação do comprimento dos frutos e das sementes e do número de septos por fruto.

Por meio de correlação simples, foram verificadas as relações entre o número de septos e comprimento de frutos, com o comprimento das sementes. Não foram considerados nesta análise 26 frutos de $B$. pulchella que apresentaram sementes danificadas por fungos e 14 frutos de $M$. somnians que apresentaram sementes inviáveis. Para cada espécie foram estimadas a taxa de predação $(\mathrm{Tp}=100 \mathrm{~Np} / \mathrm{Nv})$, 
onde Npé o número de sementes predadas e Nv o número de sementes viáveis; e a taxa de perda $(\mathrm{Tpe}=100[\mathrm{~Np}+\mathrm{Nn}] /[\mathrm{Ns}+\mathrm{n}]$; onde $\mathrm{Nn}$ é o número de sementes não viáveis, Ns o número de septos e n o número de frutos analisados. Sementes danificadas por fungos em Bauhinia pulchella foram consideradas como predadas, para o cálculo da taxa de perda.

Para Bauhinia pulchella, as diferenças no tamanho de 97 frutos de 17 plantas foram testadas pela análise de variância para um fator (Zar, 1984). Para verificar se predadores estariam escolhendo sementes de determinados tamanhos, foram tomadas ao acaso 30 sementes viáveis e 30 sementes predadas. As diferenças nas médias do comprimento entre os dois grupos foram comparadas por meio de teste t de Student (Zar 1984).

\section{Resultados}

A Tabela 1 informa os parâmetros medidos dos frutos das quatro leguminosas estudadas: tamanho do fruto (comprimento longitudinal), número de septos em cada fruto, tamanho das sementes e número de sementes viáveis por fruto. Bauhinia pulchella mostrou variação intraespecífica no tamanho dos frutos, bem como no número de sementes viáveis por fruto. Entretanto, não houve heterogeneidade entre plantas quanto ao tamanho de frutos (ANOVA, $\mathrm{F}=1,98 ; \mathrm{p}>0,05 ; \mathrm{n}=97$ ). As sementes apresentaram comprimento médio de $5,42 \mathrm{~mm}$, com um coeficiente de variação de $16,7 \%$ e possuíam cores e formas variadas (diferentes larguras e espessuras). Correlações entre comprimento de frutos e número de septos por fruto com o comprimento de sementes não foram significativas $(\mathrm{r}=0,31 ; \mathrm{p}>0,05 ; \mathrm{n}=24$ e $\mathrm{r}=0,07 ; \mathrm{p}>0,05 ; \mathrm{n}=24$, respectivamente). A média dos tamanhos de sementes predadas foi superior à de sementes não predadas $(\mathrm{t}=8,1 ; \mathrm{p}<0,05 ; \mathrm{g} .1 .=58)$. Esta tendência pode ser visualmente comprovada na Figura 1. A taxa de predação por bruquídeos foi de $11,6 \%$, a perda por ataque de fungos 43,0\%, resultando numa taxa de perda total de $64,4 \%$. Bauhinia pulchella foi a única das espécies estudadas a apresentar perdas por ataque de fungos.

Mimosa somnians apresentou as maiores variações no comprimento dos frutos (c.v. $=26,3 \%$ ), no número de septos (c.v.=39,1\%) e no número de sementes viáveis por fruto (c.v. $=38,5 \%$ ). Suas sementes possuíam formas, cores e tamanhos variáveis e apresentavam baixa dureza. A taxa de predação foi de $4,3 \%$ e a de perda $40,6 \%$. As correlações entre comprimento de frutos e número de septos por fruto com o comprimento médio de sementes não foram significativas $(r=0,18 ; p>0,05 ; n=36$ e $r=0,25$; $\mathrm{p}>0,05 ; \mathrm{n}=36$, respectivamente).

Mimosa acutistipula, espécie mais abundante na área, apresentou sementes extremamente achatadas e de formato homogêneo, cujo comprimento médio de 4,5 $\mathrm{mm}$ mostrou um coeficiente de variação de $8,9 \%$. Apenas duas sementes foram encontradas sendo predadas por larvas, resultando em uma taxa de predação de $0,7 \%$. A taxa de perda foi de $12,5 \%$. As correlações entre o número de septos e o comprimento dos frutos com o comprimento de sementes não foram significativas $(r=-0,08$; $\mathrm{p}>0,05 ; \mathrm{n}=50$ e $\mathrm{r}=0,18 ; \mathrm{p}>0,05 ; \mathrm{n}=50$, respectivamente). 
Phaseolus linearis apresentou frutos com casca resistente e sementes duras e compactas, de formato ovalado. Os coeficientes de variação relativos ao tamanho dos frutos e das sementes foram os menores dentre as espécies estudadas (10,6\% e 8,1\%, respectivamente). A taxa de predação foi de $4,1 \%$ e a de perda $14,7 \%$. A relação entre o comprimento médio de sementes com o número de septos por fruto foi significativa $(\mathrm{r}=-0,29 ; \mathrm{p}>0,05 ; \mathrm{n}=50)$, não sendo significativa para a relação entre comprimento do fruto e o comprimento médio das sementes $(\mathrm{r}=-0,01 ; \mathrm{p}>0,05 ; \mathrm{n}=50)$.

Os insetos predadores de sementes encontrados eram coleópteros Bruchidae, que apresentavam colorações crípticas. A Figura 2 mostra a relação entre tamanho de sementes e tamanho de coleópteros em Mimosa somnians, Bauhinia pulchella $e$ Phaseolus linearis. Foram, também, encontrados Hymenoptera parasitóides dos insetos de Bauhinia pulchella.

Tabela 1 - Média, desvio padrão, amplitude de variação (entre parênteses) e coeficientes de variação do tamanho e número de septos de frutos, número e tamanho de sementes de Bauhinia pulchella, Mimosa acutistipula, Mimosa somnians e Phaseolus linearis, na área de canga de Serra dos Carajás, no estado do Pará. Tamanho em milímetros

\begin{tabular}{|c|c|c|c|c|}
\hline & $\begin{array}{l}\text { Bauhinia } \\
\text { pulchella }\end{array}$ & $\begin{array}{c}\text { Mimosa } \\
\text { acutistipula }\end{array}$ & $\begin{array}{l}\text { Mimosa } \\
\text { somnians }\end{array}$ & $\begin{array}{l}\text { Phaseolus } \\
\text { linearis }\end{array}$ \\
\hline tamanho do fruto & $\begin{array}{l}127,2 \pm 17,0 \\
(90,5-170,4) \\
N=50 \\
\text { c.v. }=13,4 \%\end{array}$ & $\begin{array}{l}58,7 \pm 9,8 \\
(27,0-78,3) \\
N=50 \\
\text { c.v. }=16,7 \%\end{array}$ & $\begin{array}{l}59,0 \pm 15,5 \\
(19,1-86,6) \\
N=50 \\
\text { c.v. }=26,3 \%\end{array}$ & $\begin{array}{l}73,8 \pm 7,8 \\
(52,4-89,1) \\
N=50 \\
c . v=10,6 \%\end{array}$ \\
\hline número de septos & $\begin{array}{l}17,3 \pm 3,2 \\
(10-23) \\
N=50 \\
\text { c.v. }=18,5 \%\end{array}$ & $\begin{array}{l}6,9 \pm 1,1 \\
(5-9) \\
\mathrm{N}=50 \\
\text { c.v. }=15,6 \%\end{array}$ & $\begin{array}{l}8,3 \pm 3,2 \\
(2-17) \\
N=50 \\
\text { c.v. }=39,1 \%\end{array}$ & $\begin{array}{l}8,2 \pm 2,8 \\
(5-11) \\
\mathrm{N}=50 \\
\text { c.v. }=3,4 \%\end{array}$ \\
\hline tamanho da semente & $\begin{array}{l}5,4 \pm 0,9 \\
(3,5-7,4) \\
N=265 \\
\text { c.v. }=16,7 \%\end{array}$ & $\begin{array}{l}4,5 \pm 0,4 \\
(3,2-5,5) \\
N=287 \\
\text { c.v. }=8,9 \%\end{array}$ & $\begin{array}{l}3,8 \pm 0,6 \\
(2,5-4,9) \\
N=236 \\
\text { c.v. }=15,8 \%\end{array}$ & $\begin{array}{l}8,5 \pm 0,7 \\
(7,0-10,0) \\
N=341 \\
\text { c.v. }=8,1 \%\end{array}$ \\
\hline $\begin{array}{l}\text { número de sementes } \\
\text { viáveis }\end{array}$ & $\begin{array}{l}5,5 \pm 6,4 \\
(5-20) \\
N=277 \\
\text { c.v. }=116,0 \%\end{array}$ & $\begin{array}{l}5,9 \pm 1,4 \\
(3-8) \\
\mathrm{N}=296 \\
\text { c.v. }=23,3 \%\end{array}$ & $\begin{array}{l}8,3 \pm 3,2 \\
(2-12) \\
N=413 \\
\text { c.v. }=38,5 \%\end{array}$ & $\begin{array}{l}6,8 \pm 1,6 \\
(4-10) \\
N=341 \\
\text { c.v. }=23,5 \%\end{array}$ \\
\hline
\end{tabular}

\section{Discussão}

Se a variabilidade no tamanho e número de sementes por fruto demonstradas neste trabalho para Bauhinia pulchella e Mimosa somnians pode ser considerada um mecanismo de defesa segundo Smith (1975), como explicar as altas taxas de predação, apresentadas por estas espécies? 


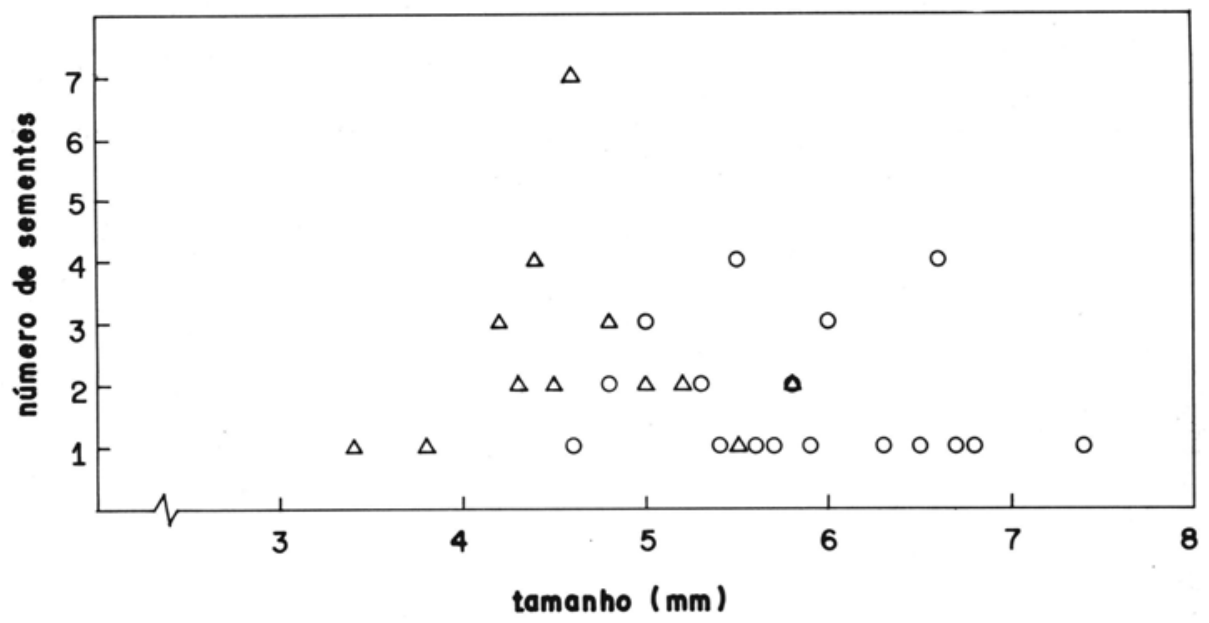

Figura 1 - Relação entre número e tamanho $(\mathrm{mm})$ de sementes predadas $(\mathrm{O})$ e não predadas $(\Delta)$ em Bauhinia pulchella coletadas na Serra Norte, Carajás, Pará.

A predação das sementes em ambas foi provocada por coleópteros Bruchidae, conhecidos por sua imunidade às substâncias tóxicas e grande especificidade às suas plantas hospedeiras (Janzen 1980), além da alta capacidade de contornar mecanismos defensivos em processos evolutivos (Center \& Johnson 1974). Como bruquídeos se alimentam, geralmente, de uma única semente (Janzen 1969), a escolha de sementes maiores pelo bruquídeo de Bauhinia pulchella pode ter implicações na quantidade de recursos necessários à sobrevivência destes insetos. A preferência por sementes maiores para predação é citada por Smith (1975) como um dos padrões pelos quais predadores interagem com sementes. Conseqüentemente, a produção de sementes de diferentes tamanhos pode significar uma adaptaçã̀o das plantas em ter sementes pequenas que escapem da predação (Janzen 1969) e possam produzir plântulas com sucesso.

É importante ressaltar que o tamanho e a dotação energética de cada semente sofrem influências de outros tipos de pressões seletivas, além da ação de predadores. De grande importância são também considerados os processos de dispersão, ajuste com agentes polinizadores (Janzen 1978; Fenner 1985), e competição entre as plântulas.

Em Mimosa somnians, a maior proporção de perdas não se deu à predação, mas ao grande número de sementes inviáveis por ela produzidas. A imprevisibilidade do número de sementes viáveis em cada fruto, neste caso, poderia consistir num mecanismo de defesa, uma vez que impede a otimização da quantidade de ovos deixados pelo predador em cada fruto. Oshima et al. (1973) e Koslowski et al. (1983) verificaram que algumas espécies de bruquídeos regulam o número de ovos depositados em cada 
fruto, por meio de marcações químicas que evitam repetidas oviposições num mesmo substrato. Assim, a distribuição de ovos pode ser crítica para o sucesso reprodutivo de predadores, uma vez que frutos representariam uma unidade discreta de recursos, não havendo possibilidades de larvas intercambiarem de um fruto para outro (Lewinson 1980).

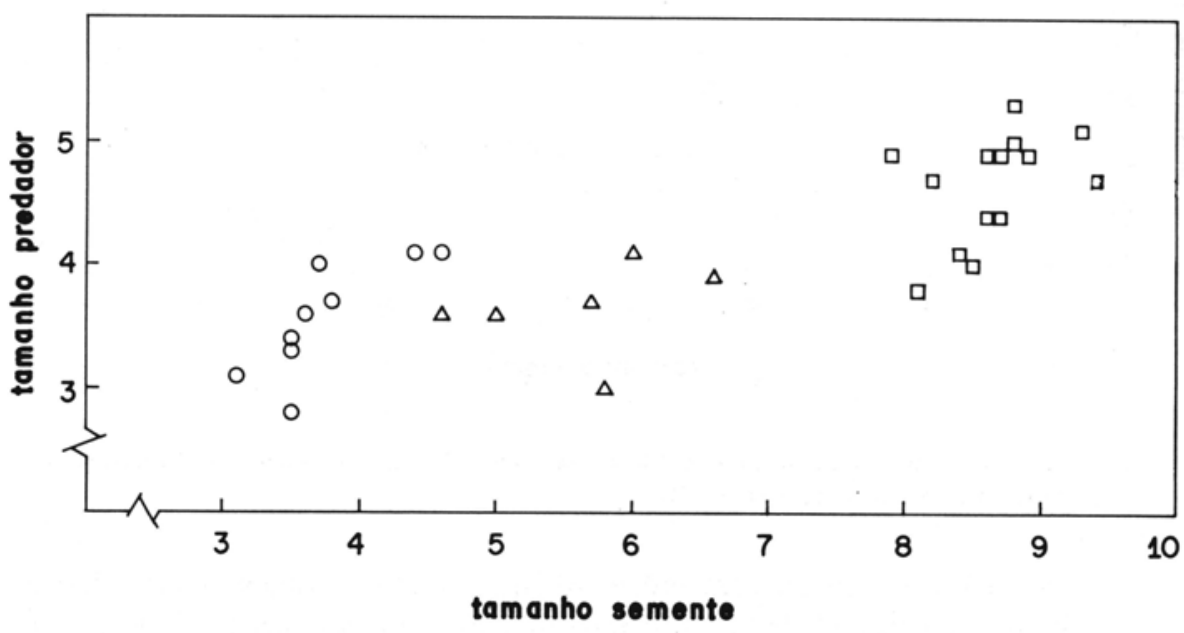

Figura 2 - Relação entre tamanho de predadores e tamanho de sementes (mm) de Mimosa somnias (O), Bauhinia pulchella $(\Delta)$ e Phaseolus linearis $(\square)$, coletadas na Serra Norte, Carajás, Pará.

Mimosa acutistipula, espécie mais freqüente, apresentou a menor taxa de predação dentre as espécies analisadas. $\mathrm{O}$ fato de as sementes serem extremamente achatadas parece limitar a ação de predadores, impedindo-os de se desenvolverem em seu interior (Bridwell 1918 apud Janzen 1969). A dependência entre o tamanho do predador e o tamanho da semente, proposta para as outras espécies neste estudo, viria corroborar a hipótese de Janzen (1969) de que a redução na quantidade de nutrientes em sementes constitui uma estratégia de defesa contra predadores.

Em Phaseolus linearis a alta resistência da casca à penetração, aliada à dureza de suas sementes podem estar dificultando o acesso dos predadores ao interior do fruto e das sementes. É possível que a relativa eficácia deste mecanismo de defesa possa ter relação com a pequena variabilidade do número e tamanho de sementes produzidas em cada fruto.

\section{Agradecimentos}

Agradeço aos professores Carlos Frederico Duarte da Rocha, João Vasconcellos-Neto e Marli Aparecida Ranal as sugestões dadas na leitura prévia do manuscrito. 


\section{Referências bibliográficas}

Center, T. D. \& Johnson, C. D. 1974. Coevolution of some seed beetles (Coleoptera: Bruchidae) and their hosts. Ecology 55:1096-1103.

Connell, J. H. 1970. On the role of natural enemies in preventing competitive exclusion in some marine animals and in rain forest trees In: Denboer, P. V. \& Gradwell, G. R. (eds.) Dynamics of populations. Wageningen, Centre for Agricultural Publishing and Documentation. p.289-312.

Fenner, M. 1985. Seed ecology. London, Chapman and Hall. 151 p.

Fernandes, G. W. \& Whitham, T.G. 1989. Selective abscision by Juniperus monosperma as an induced defense against predators. Amer. Midl. Nat. 121:389-392.

Janzen. D. H. 1969. Seed-eaters versus seed size, number, toxicity and dispersal. Evolution 23:1-27.

Janzen, D. H. 1970. Herbivores and the number of tree species in tropical forest. Am. Nat. 104:501-528.

Janzen, D. H. 1971. Seed predation by animals. Ann. Rev. Ecol. Syst. 2:465-492

Janzen, D. H. 1978. Inter- and intra-crop variation in seed weigth of Costa Rican Ateleia herbert-smithii Pitt. (Leguminosae). Brenesia 14/15:311-323.

Janzen, D. H. 1980. Specificity of seed-attacking bettles in a Costa Rican deciduous forest. J. Ecol. 68:929-952.

Koslowski, M. W., Lux, S. \& Dmoch, J.. 1983. Oviposition behaviour and pod marking in the cabbage seed weevil Ceutorhynchus assimilis. Entomol. Exp. Appl. 34:277-282.

Lewinsohn, T. M. 1980. Predação de sementes em Hymenaea (Leguminosae: Caesalpinioideae): aspectos ecológicos e evolutivos. Campinas: Universidade Estadual de Campinas. Dissertação de Mestrado.

Oshima, K., Honda, H. \& Yamamoto, I. 1973. Isolation of an oviposition marker from azuki bean weevil, Callosobruchus chinensis (L.). Agr. Biol. Chem. 37:2679-2680.

Secco, R. S. \& Mesquita, A. L. 1983. Notas sobre a vegetação de canga de Serra Norte. Bol. Mus. Par. Emílio Goeldi (Botânica) 59:1-13.

Smith, C.C. 1975. The coevolution of plants and seed predators. in: Gilbert, L. E. \& Raven, P. H. (eds.) Coevolution of animals and plants. Austin, Texas University Press. p. 53-77.

Wilson, D. E. \& Janzen, D. H. 1972. Predation on Scheelea palm seeds by bruchid beetles: seed density and distance from the parent palm. Ecology 53:954-959.

Zar, J. H. 1984. Biostatistical analysis. New Jersey, Prentice-Hall. 